\title{
Índices de vegetação para a estimativa do índice de área foliar em plantios clonais de Eucalyptus saligna Smith
}

\author{
Vegetation indices for the leaf area index estimation in clonal plantations of \\ Eucalyptus saligna Smith
}

\author{
Rute BergerI, José Antônio Aleixo da Silva ${ }^{\mathrm{II}}$, Rinaldo Luiz Caraciolo Ferreira ${ }^{\mathrm{III}}$, Ana \\ Lúcia Bezerra Candeias ${ }^{\mathrm{IV}}$, Rafael RubilarV
}

\begin{abstract}
Resumo
Procurou-se estabelecer a relação entre o Índice de área foliar no campo (IAF) de plantios clonais de Eucalyptus saligna Smith e três diferentes Índices de Vegetação (IV) obtidos de uma imagem Landsat 8/OLI: Índice de Vegetação da Diferença Normalizada (Normalized Difference Vegetation Index, em inglês - NDVI), Índice da Razão Simples (Simple Ratio Index - SRI) e Índice de Vegetação Ajustado para o Solo (Soil Adjusted Vegetation Index - SAVI), com o objetivo de selecionar o melhor estimador do IAF por sensoriamento remoto $\left(\mathrm{IAF}_{\mathrm{SR}}\right)$, obtendo assim a espacialização IAF nos talhões. O IAF foi obtido utilizando o equipamento LAI2000 e seu comportamento foi analisado em diferentes idades. Os índices de vegetação foram obtidos por meio de aritmética das bandas 4 e 5 do sensor. A análise de regressão linear simples foi utilizada para ajustar o modelo de estimativa de IAFSR (IAFSRi $=\beta_{0}+\beta_{1} \cdot I_{\mathrm{i}}+\varepsilon_{\mathrm{i}}$ ), sendo os critérios de escolha as estatísticas de R2aj\%, Syx\% e análise de resíduos. Os resultados mostraram que o índice que melhor estimou o $\mathrm{IAF}_{\mathrm{SR}}$ foi o SRI $\left(\mathrm{IAF}_{\mathrm{SR}}=-5,6159+0,9716\right.$. SRI), com $\mathrm{R}_{\mathrm{aj}}^{2} \%=67,0$ e $\mathrm{S}_{\mathrm{yx}} \%=12,5$. Todos os modelos ajustados mostraram tendência em sobrestimar o IAF em valores inferiores a dois e subestimar o IAF a partir de valores superiores a 3,5 (NDVI e SRI) e superiores a três para SAVI. As equações obtidas para as diferentes idades não produziram melhora nas estimativas de $\mathrm{IAF}_{\mathrm{SR}}$.
\end{abstract}

Palavras-chave: Análise de regressão; Índice da Razão Simples (SRI); LAI-2000; Landsat 8/OLI

\begin{abstract}
The relationship between the ground Leaf area index (LAI $)$ from clonal plantations of Eucalyptus saligna Smith and three different vegetation indices (VI): Normalized Difference Vegetation Index (NDVI), Simple Ratio Index (SRI) and Soil Adjusted Vegetation Index (SAVI) was evaluated in order to select the best IV to estimate the IAF by remote sensing $\left(\mathrm{LAI}_{\mathrm{RS}}\right)$, and obtaining the spatial distribution of LAI in the stands. $\mathrm{LAI}_{\mathrm{g}}$ was measured using LAI-2000 and its behavior was examined at different ages. The vegetation indices were obtained from a Landsat 8/OLI through the arithmetic of the bands 4 and 5 . The linear regression analysis was used to adjust the model LAIRS (LAIRSi $=\beta_{0}+\beta_{1} . \mathrm{IV}_{\mathrm{i}}+\varepsilon_{\mathrm{i}}$ ), and the criteria for selecting the best equation were the statistics R2adj\%, Syx \% and residual analysis. The results showed that the best vegetation index to estimate $\mathrm{IAF}_{\mathrm{SR}}$ was SRI $\left(\mathrm{LAI}_{\mathrm{RS}}=-5.6159+0.9716\right.$. SRI ), resulting $\mathrm{R}^{2}{ }_{\text {adj }} \%=67.0$ and $S_{\mathrm{yx}}=12.5 \%$. The results of all adjusted models tended towards overestimation of LAI in values lower than two and underestimation in values above 3.5 (NDVI e SRI) and above three for SAVI. The equations for different ages produced no improvement in $\mathrm{LAI}_{\mathrm{RS}}$.
\end{abstract}

Keywords: Regression analysis; Simple Ratio Index (SRI); LAI-2000; Landsat 8/OLI

\footnotetext{
Engenheira Florestal, Dra., Professora Adjunta do Departamento de Ciência Florestal, Universidade Federal Rural de Pernambuco, Rua Dom Manuel de Medeiros, s/n, CEP 52171-900, Recife (PE), Brasil. rute.berger@ufrpe.br (ORCID: 0000-0002-2079-0156)

Engenheiro Agrônomo, PhD, Professor Titular do Departamento de Ciência Florestal, Universidade Federal Rural de Pernambuco, Rua Dom Manuel de Medeiros, s/n, CEP 52171-900, Recife (PE), Brasil. jaaleixo@uol.com.br (ORCID: 0000-0003-0675-3524)

III Engenheiro Florestal, Dr., Professor Titular do Departamento de Ciência Florestal, Universidade Federal Rural de Pernambuco, Rua Dom Manuel de Medeiros, s/n, CEP 52171-900, Recife (PE), Brasil. rinaldo.ferreira@ufrpe.br (ORCID: 0000-0001-7349-6041)

Engenheira Eletricista, Dra., Professora Associada do Departamento de Engenharia Cartográfica, Universidade Federal de Pernambuco, Av. Prof. Moraes Rego, 1235, CEP 50.670-901, Recife (PE), Brasil. analucia@ufpe.br (ORCID: 0000-0002-9021-7603)

Engenheiro Florestal, PhD., Professor Associado do Departamento de Silvicultura, Universidad de Concepción, Victoria, 63, Casilla 160-C, Concepción, Chile. rafaelrubilar@udec.cl (ORCID: 000-0002-4929-7613)
} 


\section{Introdução}

No manejo florestal, o índice de área foliar (IAF) pode ser usado como indicador da produtividade florestal, sendo considerado como um fator crítico na gestão de florestas (HERNÁNDEZ et al., 2014) e de fácil interpretação da capacidade produtiva dos sítios (CANNELL, 1989). Definido como a quantidade de área foliar unilateral por metro quadrado de superfície $\left(\mathrm{m}^{2} \cdot \mathrm{m}^{-2}\right)$, é considerado o fator mais importante na absorção de luz pelas copas e explica boa parte da variação da produção vegetal (CANNELL, 1989). O IAF é influenciado pela estrutura da copa, ramos, entre outros, sendo que igual valor de IAF pode corresponder a diferentes tipos florestais e idades (BINKLEY et al., 2013), dependendo da espécie, sítio, condições climáticas (sazonalidade) e manejo (JONCKHEERE et al., 2004).

O IAF é positivamente relacionado com a produtividade florestal (JONCKHEERE et al., 2004; ÁLVAREZ et al., 2013; BERGER, 2014), sendo encontradas boas correlações entre IAF e produção volumétrica (BERGER et al., 2009; FOREST NUTRITION COOPERATIVE, 2007), além de resultados positivos no uso de regressões para estimar variáveis de produtividade florestal, tais como altura dominante, volume e biomassa (ARIAS; CALVO-ALVARADO; DOHRENBUSCH, 2007). Neste sentido, setores com baixo IAF poderiam ser identificados e potencializados, e mapas de IAF poderiam ser utilizados para recomendações silviculturais (COLOMBO et al., 2003).

O IAF pode ser obtido de várias maneiras, mais detalhadamente descritas por Jonckheere et al. (2004). Os métodos diretos de medição de IAF são teoricamente mais precisos que os semidiretos e que os indiretos. Entretanto, os métodos diretos consomem muito tempo em sua determinação, tornando-os praticamente inviáveis para seu uso em grande escala. Por outro lado, os métodos indiretos são de mais fácil utilização, sendo que normalmente os métodos óticos são os preferidos, por sua relativa rapidez e precisão. Neste caso, o LAI-2000 Plant Canopy Analyser é o instrumento mais comumente utilizado para estimar a quantidade de radiação difusa que se infiltra por meio das copas, sendo a maneira mais prática e simples para obter estimativas de IAF em florestas de rápido crescimento de forma instantânea (ARIAS; CALVO-ALVARADO; DOHRENBUSCH, 2007). Entretanto, a aplicação desses métodos em larga escala é onerosa, pois demanda muito tempo e o uso de equipamentos sofisticados (HERNÁNDEZ et al., 2014).

Devido à sua importância em muitos processos ecológicos, quantidade significativa de estudos tem sido realizada para estimar o IAF em média e larga escala (ARIAS; CALVOALVARADO; DOHRENBUSCH, 2007; BERGER, 2014; FLORES et al., 2006). As ferramentas de sensoriamento remoto (SR) vêm sendo utilizadas para estimar a distribuição espacial e temporal de IAF (BERGER et al., 2009; BERGER, 2014; CHEN; CIHLAR, 1996; FLORES et al., 2006; HOUBORG; ANDERSON; DAUGHTRY, 2009), havendo forte relação com os índices de vegetação (IV) de sensores de plataformas orbitais, gerando relações lineares significativas (CHEN; CIHLAR, 1996; FLORES et al., 2006; FOREST NUTRITION COOPERATIVE, 2007; BERGER, 2014), proporcionando um caminho singular para obter a distribuição de IAF em áreas extensas (CHEN; CIHLAR, 1996).

Os IV são medidas radiométricas adimensionais, geradas por meio de transformações matemáticas, que se valem das propriedades multiespectrais dos sensores remotos. Os IV têm buscado explorar as propriedades espectrais da vegetação (folhas e outros componentes fotossinteticamente ativos), principalmente nas regiões do visível e do infravermelho próximo do espectro eletromagnético. Sua fundamentação reside no comportamento antagônico da reflectância da vegetação nas regiões mencionadas, com o qual pretendem indicar a abundância relativa e a atividade da cobertura vegetal (PONZONI; SHIMABU KURO, 2010; JENSEN, 2011).

Dentre os diversos índices de vegetação apresentados por Ponzoni e Shimabukuro (2010) e Jensen (2011), destacam-se o Índice de Razão Simples (Simple Ratio Index - SRI) e o Índice de Vegetação da Diferença Normalizada (Normalized Difference Vegetation Index - NDVI) como os mais amplamente utilizados para caracterizar o IAF e a biomassa de formações florestais 
(FLORES et al., 2006; JENSEN, 2011). O Índice de Vegetação Ajustado para o Solo (Soil Adjusted Vegetation Index - SAVI), cuja introdução de uma constante busca minimizar o efeito do solo, também tem sido utilizado para formações florestais (PONZONI; SHIMABUKURO, 2010).

O SRI é provavelmente o primeiro IV utilizado (JACKSON; HUETE, 1991; PONZONI; SHIMABUKURO, 2010) e baseia-se no princípio de que as folhas absorvem relativamente mais radiação eletromagnética na região do vermelho (visível) do que na região do infravermelho. Sendo assim, quanto maior a quantidade de folhas no dossel, maior será o valor da razão. O NDVI é provavelmente o mais popular, sendo amplamente aplicado para dados de sensores remotos do satélite Landsat (PONZONI; SHIMABUKURO, 2010). O SAVI tem sido utilizado para diminuir o efeito do solo em condições de vegetação com cobertura média (JENSEN, 2011).

Huete, Jackson e Post (1985) analisaram a sensibilidade dos IV em relação ao solo e verificaram que essa é maior em dosséis com cobertura média.

Assim como os demais IV, o SRI e o NDVI apresentam vantagens e desvantagens e sua interpretação deve levar em consideração vários fatores limitantes, tais como: pontos de saturação, interferência atmosférica, entre outros (PONZONI; SHIMABUKURO, 2010). Jackson e Huete (1991) comentam que os IV podem ser influenciados pelo teor de umidade, rugosidade, sombra ou por diferentes teores de matéria orgânica no solo.

Vários trabalhos abordam a relação dos IV obtidos por SR com os parâmetros biofísicos das florestas, sendo destacados aqueles que se referem IAF (CHEN; CIHLAR, 1996; HOUBORG; ANDERSON; DAUGHTRY, 2009; JENSEN, 2011; SONG, 2013).

Os objetivos deste trabalho foram avaliar as relações entre o IAF medido no campo (IAF ), em idades variando entre três e oito anos, com os diferentes índices de vegetação (IV) obtidos de uma imagem Landsat 8/OLI, procurando estabelecer modelos de predição de IAF em função de IV de SR para a geração de um mapa de IAF estimado por SR $\left(\mathrm{IAF}_{\mathrm{SR}}\right)$ para plantios clonais de Eucalyptus saligna Smith.

\section{Material e método}

\section{Local de estudo e características dos plantios selecionados}

A área de estudo está localizada no município de Barra do Ribeiro, Rio Grande do Sul, Brasil. Os dados foram coletados no Horto Florestal Barba Negra (HFBN), de propriedade da empresa Celulose Rio-grandense - Grupo CMPC, cuja localização geográfica está situada entre os paralelos $30^{\circ} 27^{\prime} 56,802^{\prime \prime}$ S e $30^{\circ} 26^{\prime} 33,803^{\prime \prime} \mathrm{S}$, e entre os meridianos $51^{\circ} 16^{\prime} 06,079^{\prime \prime} \mathrm{W}$ e $51^{\circ} 05^{\prime} 46,049$ ” W (Datum WGS84). Conforme o sistema de classificação Köppen-Geiger, o clima da região é do tipo Cfa (temperado, verão quente e sem estação seca) (PEEL; FINLAYSON; MCMAHON, 2007). Na Figura 1 são mostrados os dados de precipitação e temperatura mensal obtidos em 2013 na estação meteorológica instalada no HFBN. A precipitação total foi de $1.378,21 \mathrm{~mm}$, com temperatura máxima média do mês mais quente de $24,1^{\circ} \mathrm{C}$ e temperatura mínima média do mês mais frio de $11,3^{\circ} \mathrm{C}$. O relevo do local varia de suave ondulado a plano, predominando este último. Os solos da região são de formação recente, tais como argissolos, gleissolos, neossolos e planossolos.

Foram selecionados talhões comerciais com condições internas homogêneas de idade, manejo e espaçamento. O material genético selecionado para a avaliação foi um clone de Eucalyptus saligna Smith, plantado sob diferentes espaçamentos $(3,00 \mathrm{~m} \times 3,00 \mathrm{~m}$, $3,00 \mathrm{~m} \times 2,50 \mathrm{~m}$ e $3,50 \mathrm{~m} \times 2,14 \mathrm{~m}$ ), diferentes sítios e com idades variando entre três e oito anos. 


\section{Figura 1 - Dados de precipitação e temperatura da estação meteorológica do Horto Florestal Barba Negra durante o ano 2013.}

Figure 1 - Precipitation and temperature from the meteorological station of the 'Barba Negra' Farm, during 2013.

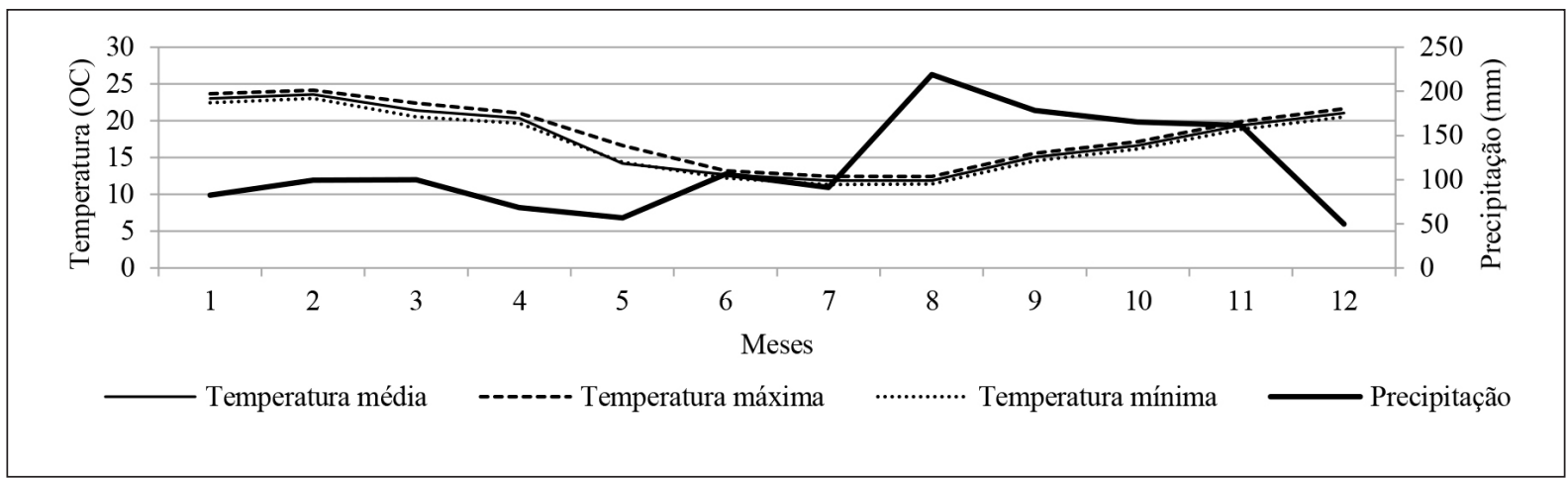

Fonte: Dados meteorológicos do HFBN, CMPC Celulose Rio-grandense (2013)

\section{Medição do Índice de Área Foliar no campo}

A medição do Índice de Área Foliar no campo ( $\mathrm{IAF}_{\mathrm{c}}$ ) foi realizada durante os meses de março e abril de 2013, utilizando o equipamento de medição indireta de IAF, o Li-Cor LAI-2000 Plant Canopy Analyser (LI-COR, 1992). Conforme proposto pelo fabricante, as medições foram realizadas sob condições de radiação difusa, nas primeiras horas da manhã e no final da tarde. Utilizou-se o método dos dois sensores, com um sensor de referência coletando dados em uma área livre de vegetação (campo aberto) e outro sensor de medição fazendo as coletas sob o dossel nas unidades amostrais (UA). Ambos os sensores foram posicionados e orientados na mesma direção. Como as UA eram de tamanho reduzido $\left(400 \mathrm{~m}^{2}\right)$ e a coleta foi realizada por operadores, o campo de visualização azimutal do sensor foi restringido a 45\%. Todas as medições com o equipamento foram tomadas entre as linhas de plantio e a $1,30 \mathrm{~m}$ do solo, para reduzir o efeito do sub-bosque.

Foram consideradas as medições de $\mathrm{IAF}_{\mathrm{c}}$ em $40 \mathrm{UA}$, pertencentes a 11 talhões $(246,33$ ha). Em cada UA foram tomadas em média 30 medições de IAF, cobrindo toda a superfície da parcela. O valor do IAF de cada UA foi obtido pela média dessas medições, obtido diretamente pelo equipamento.

\section{Obtenção dos Índices de Vegetação}

Para a análise foi utilizada uma imagem orbital obtida pelo sensor Operational Land Imager (OLI), a bordo do satélite Landsat 8, gerada pelo United States Geological Survey (UNITED STATES GEOLOGICAL SURVEY, 2013). Essa foi disponibilizada gratuitamente pela página web da USGS, em formato GeoTIFF (georreferenciada), com $30 \mathrm{~m}$ de resolução espacial. As especificações da imagem utilizada estão descritas na Tabela 1.

Os números digitais (ND) da imagem foram convertidos para radiância (Li), utilizando os valores de ganho e offset disponíveis no arquivo de metadados da imagem. Posteriormente, a radiância foi convertida para reflectância $\left(\rho_{\mathrm{i}}\right)$ no topo da atmosfera, conforme a expressão (1) (PONZONI; SHIMABUKURO, 2010). O software utilizado para o tratamento dos dados foi o ENVI 5.1 (ENVI 5.1, 2014), versão trial 


$$
\rho_{i}=\frac{\pi \cdot L_{i} \cdot d^{2}}{E_{\text {sun }} \cdot \cos \theta}
$$

Em que: $\rho_{\mathrm{i}}=$ fluxo radiante ou reflectância no topo da atmosfera, para a banda i; $\mathrm{L}_{\mathrm{i}}=$ radiância aparente (Watts. $\mathrm{m}^{-2} \cdot \mathrm{sr}^{-2} \cdot \mu \mathrm{m}^{-1}$ ), para a banda i; $\mathrm{d}=$ distância Sol-Terra em unidades astronômicas; $\mathrm{E}_{\text {sun }}=$ Irradiância média do sol no topo da atmosfera $\left(\right.$ Watts $\left.\mathrm{m}^{-2} \cdot \mathrm{sr}^{-2} \cdot \mu \mathrm{m}^{-1}\right) ; \theta=$ ângulo solar zenital.

Para a obtenção dos índices de vegetação (IV), após o pré-processamento da imagem, foram realizadas operações matemáticas utilizando a banda 4 (fluxo radiante refletido no vermelho) e a banda 5 (fluxo radiante refletido no infravermelho próximo). No sensor OLI, essas bandas espectrais operam entre o comprimentos de onda $0,64-0,67 \mu \mathrm{m}$ e $0,85-0,88 \mu \mathrm{m}$, respectivamente, para banda 4 e banda 5 (UNITED STATES GEOLOGICAL SURVEY, 2013). A função Math Band do ENVI 5.1 foi utilizada para realizar as operações. Após o processamento dos dados, os arquivos foram exportados para o software ArcMap (Versão 10.1).

\section{Tabela 1 - Especificações da imagem do sensor OLI/Landsat 8 utilizada para a estimativa dos Índices de Vegetação, em maio/2013.}

Table 1 - Specifications of the Landsat 8/OLI image used for the vegetation index estimations, May-2013.

\begin{tabular}{lc}
\hline \multicolumn{2}{c}{ Especificações da imagem OLI/LANDSAT 8 } \\
\hline Órbita/Ponto & $221 / 81$ \\
Data & $26 / 05 / 2013$ \\
Hora (TMG) & $13: 20: 46$ \\
Azimute Solar & $33,44^{\circ}$ \\
Elevação Solar & $30,87^{\circ}$ \\
Qualidade da Imagem & $9^{*}$ \\
Sistema Geodésico de Referência & WGS84 \\
\hline
\end{tabular}

Fonte: USGS (2013)

As expressões (2), (3) e (4) representam como foram obtidos o SRI, NDVI e SAVI, respectivamente (JENSEN, 2011):

$$
\begin{aligned}
& \text { NDVI }=\left(\rho_{\text {IVP }}-\rho_{\mathrm{V}}\right) /\left(\rho_{\mathrm{IVP}}+\rho_{\mathrm{V}}\right) \\
& \mathrm{SRI}=\rho_{\mathrm{IVP}} / \rho_{\mathrm{V}} \\
& \mathrm{SAVI}=\left[\left(\rho_{\mathrm{IVP}}-\rho_{\mathrm{V}}\right) /\left(\rho_{\mathrm{IVP}}+\rho_{\mathrm{V}}+\mathrm{L}\right)\right]^{*}(1+\mathrm{L})
\end{aligned}
$$

Em que: $\mathrm{p}_{\mathrm{v}}, \mathrm{p}_{\mathrm{IVP}}$ referem-se ao fluxo radiante refletido no vermelho (visível) e ao fluxo radiante refletido no infravermelho próximo, respectivamente; $L$ é a constante que minimiza o efeito do solo. Para a constante L do SAVI, foi adotado o valor de 0,5 para uma densidade de vegetação média, conforme Huete (1988). 
Relação $\mathrm{IAF}_{c}$ e índices de vegetação

Os valores de $\mathrm{IAF}_{\mathrm{c}}$ foram relacionados aos valores dos IV em cada UA, sendo esses resultantes de uma média entre os oito pixels vizinhos ao pixel que continha o centro da UA e este último, totalizando nove pixels. Esta metodologia foi adotada porque as coordenadas UTM das UA foram registradas com uso de GPS navegador, cujos erros podem ser superiores a $5 \mathrm{~m}$, o que não assegura sua localização exata no campo. Metodologia semelhante foi utilizada por Salvador e Pons (1998) e Forest Nutrition Cooperative (2007).

Para reduzir a variabilidade entre a relação $\mathrm{IAF}_{c}$ e IV foram eliminados todos os pontos de medição cujo coeficiente de variação entre IV dos nove pixels tenha sido maior que $10 \%$. Também foram eliminados todos os pontos localizados em áreas limites, tais como bordas, caminhos, corpos de água, ou outros usos do solo que pudessem interferir nas estimativas de $\mathrm{IAF}_{\mathrm{SR}}$.

A análise de regressão linear simples foi utilizada para estabelecer e selecionar equações para estimar o IAF em função dos diferentes IV. Os critérios para a seleção da melhor equação foram as estatísticas coeficiente de determinação $\left(\mathrm{R}^{2}{ }_{\mathrm{aj}} \%\right)$, erro padrão da estimativa $\left(\mathrm{S}_{\mathrm{yx}} \%\right)$ e distribuição gráfica de resíduos, bem como a significância dos coeficientes da equação ( $\mathrm{F}=5 \%$ ). Adicionalmente foi realizada uma análise semelhante considerando separadamente a idade de plantio, buscando verificar se algum dos IV poderia estimar o IAF com maior precisão em uma idade específica. Os dados dos plantios de oito anos foram analisados juntamente aos dados dos plantios de seis anos por haver apenas duas amostras.

Definida a equação, a mesma foi utilizada para estimar o IAF de cada pixel em função dos respectivos IV da imagem, utilizando a função Raster Calculator do ArcGIS 10.1, gerando uma imagem $\mathrm{IAF}_{\mathrm{SR}}$.

\section{Resultados e discussão}

\section{Caracterização do Índice de Área Foliar medido no campo}

Os resultados IAF $_{c}$ obtidos com o LAI-2000 mostram que em todas as idades avaliadas, houve ampla variação entre as UA, com valores entre 1,30 e 4,01 (Tabela 2).

Tabela 2 - Estatística descritiva para índice de área foliar medido no campo $\left(\mathrm{IAF}_{\mathrm{c}}\right)$ de um clone de Eucalyptus saligna Smith em diferentes idades.

Table 2 - Summary statistics for ground leaf area index (LAI $)$ of a Eucalyptus saligna Smith clone at different ages.

\begin{tabular}{lcccc}
\hline & \multicolumn{5}{c}{ Idade (anos) } \\
\cline { 2 - 5 } $\mathbf{I A F}_{\mathbf{c}}$ & $\mathbf{3}$ & $\mathbf{5}$ & $\mathbf{6}$ & $\mathbf{8}$ \\
\hline Média & 3,28 & 2,66 & 2,51 & 1,37 \\
Mínima & 2,43 & 1,87 & 1,69 & 1,30 \\
Máxima & 4,01 & 3,24 & 2,85 & 1,44 \\
Coeficiente de Variação (\%) & 16,9 & 14,37 & 13,94 & 7,23 \\
Amostras & 10 & 17 & 11 & 2 \\
\hline
\end{tabular}

Fonte: Autores (2015) 
Quando analisados por idade, os valores de $\mathrm{IAF}_{c}$ variaram entre 2,43 e 4,01 aos três anos, entre 1,87 e 3,24 aos cinco anos, entre 1,69 e 2,85 aos seis anos e entre 1,30 e 1,44 aos oito anos. A menor amplitude de $\operatorname{IAF}_{c}$ foi observada aos oito anos $(0,14)$ e a maior aos três anos $(1,58)$. Resultados semelhantes foram obtidos por Xavier, Soares e Almeida (2002) estudando clones híbridos de Eucalyptus grandis (15 e 84 meses) no Norte do estado do Espírito Santo, por Almeida et al. (2010), avaliando plantios clonais de Eucalyptus sp. entre três e seis anos de idade e por Rody et al. (2014) estudando clones híbridos de Eucalyptus grandis x Eucalyptus urophylla. Os maiores valores de $\mathrm{IAF}_{\mathrm{c}}$ foram encontrados em plantios jovens e os menores em plantios mais velhos.

\section{Índice de Área Foliar em função dos índices de vegetação}

Os resultados obtidos mostram que o melhor ajuste foi obtido quando utilizado o SRI como variável independente para estimar o IAF (Equação 2, Tabela 3). A variação total explicada pela regressão foi de $\mathrm{R}_{\mathrm{aj}}^{2} \%=67,0 \%$. Essa equação também gerou a menor dispersão percentual entre os dados observados e estimados pela regressão $\left(\mathrm{S}_{\mathrm{yx}} \%\right)$, com 12,5\%.

Resultados satisfatórios para as estimativas de IAF em função do SRI também foram encontrados por outros estudos (FLORES et al., 2006; FOREST NUTRITION COOPERATIVE, 2007) e provavelmente resultam das características da vegetação, pois o SRI é altamente sensível às variações de biomassa abundante para formações florestais (JENSEN, 2011).

O NDVI (Equação 1, Tabela 3) gerou um bom ajuste de regressão $\left(\mathrm{R}_{\mathrm{aj}}^{2} \%=65,4\right)$, ligeiramente inferior ao SRI. Entretanto, o $\mathrm{S}_{\mathrm{yx}} \%$ mostrou maior dispersão entre os dados observados e estimados (16,2\%). O NDVI é apontado como um índice mais sensível para condições de baixa biomassa, tais como biomas áridos ou semiáridos (FAN et al., 2009; JENSEN, 2011), e plantios com menores valores de IAF. Além disso, pode sofrer a influência de pontos de saturação nas condições de biomassa abundante, tais como as florestas, não caracterizando adequadamente o IAF (PONZONI; SHIMABUKURO, 2010; JENSEN, 2011).

A utilização do SAVI para estimar IAF resultou em baixo $\mathrm{R}_{\mathrm{aj}}^{2} \%(33,4 \%)$ e o maior $\mathrm{S}_{\mathrm{yx}} \%$ $(17,8 \%)$ (Equação 3, Tabela 3). Apesar da significância do coeficiente angular $\mathbb{\bigotimes}_{1}$ (Prob $>F=5,58.10^{-}$ ${ }^{5}$ ), esta equação apresentou o pior desempenho quando comparada às demais equações. Outras análises também apontam resultados de baixo desempenho (COLOMBO et al., 2003; BULCOCK; JEWITT, 2010), e outras sem diferença em relação aos outros IV (LE MAIRE et al., 2011).

\section{Tabela 3 - Estatísticas e parâmetros de regressão para estimar o índice de área foliar (IAF) em função de Índice de Vegetação da Diferença Normalizada (NDVI), Índice da Razão Simples (SRI) e Índice de Vegetação Ajustado para o Solo (SAVI) de uma imagem Landsat 8.}

Table 3 - Summary statistics for models fitted in the development of a relationship between leaf area index and normalized difference vegetation index (NDVI), (b) simple ratio index (SRI) and (c) soil adjusted vegetation index, obtained from Landsat 8/OLI scene.

\begin{tabular}{|c|c|c|c|c|c|c|}
\hline \multirow[t]{2}{*}{ Equação } & \multirow{2}{*}{$\begin{array}{c}\text { Índice de Vegetação } \\
\text { (variável independente) }\end{array}$} & \multicolumn{2}{|c|}{$\begin{array}{l}\text { Parâmetros do modelo* } \\
\quad \text { (coeficientes) }\end{array}$} & \multirow{2}{*}{$\begin{array}{c}\text { Prob }>\text { F } \\
\left(\mathbf{B}_{1}\right)\end{array}$} & \multirow{2}{*}{$\mathbf{R}_{a j}^{2} \%$} & \multirow{2}{*}{$S_{y x} \%$} \\
\hline & & $\mathbf{B}_{0}$ & $\mathrm{~B}_{1}$ & & & \\
\hline 1 & NDVI & $-32,2073$ & 44,1954 & $9,54 \cdot 10^{-11 * *}$ & 65,4 & 16,2 \\
\hline 2 & SRI & $-5,6159$ & 0,9716 & $6,78 \cdot 10^{-11 * *}$ & 67,0 & 12,5 \\
\hline 3 & SAVI & $-3,7866$ & 18,2500 & $5,58 \cdot 10^{-5 * *}$ & 33,4 & 17,8 \\
\hline
\end{tabular}

Fonte: Berger $(2014){ }^{*} \mathrm{IAF}=\beta_{0}+\beta_{1} . \mathrm{IV}+\varepsilon_{\mathrm{i}} ;$ Prob $>\mathrm{F}=$ valor de significância; ${ }^{* *}$ Significativo para $\mathrm{F}=5 \% ; \mathrm{R}_{\mathrm{aj}}^{2} \%=$ Coeficiente de Determinação, em percentagem; $S_{y x} \%=E r r o$ padrão da estimativa, em percentagem. 
A análise realizada por idade resultou em algumas diferenças de desempenho dos IV avaliados (Tabela 4), em relação ao modelo geral que incluiu todas as medições (Tabela 3).

Tabela 4 - Estatísticas e parâmetros de regressão para o índice de área foliar (IAF) em função de Índice de Vegetação da Diferença Normalizada (NDVI), Índice da Razão Simples (SRI) e Índice de Vegetação Ajustado para o Solo (SAVI), em diferentes idades.

Table 4 - Summary statistics for models fitted in the development of a relationship between leaf area index and normalized difference vegetation index (NDVI), (b) simple ratio index (SRI) and (c) soil adjusted vegetation index (SAVI), for different ages.

\begin{tabular}{|c|c|c|c|c|c|c|c|}
\hline \multirow{2}{*}{$\begin{array}{l}\text { Índice de Vegetação } \\
\text { (variável independente) }\end{array}$} & \multirow[t]{2}{*}{ Equação } & \multirow{2}{*}{$\begin{array}{l}\text { Idade } \\
\text { (anos) } \\
\end{array}$} & \multicolumn{2}{|c|}{$\begin{array}{c}\text { Parâmetros do modelo* } \\
\text { (coeficientes) }\end{array}$} & \multirow{2}{*}{$\begin{array}{c}\text { Prob }>\mathbf{F} \\
\left(\mathbf{B}_{1}\right)\end{array}$} & \multirow{2}{*}{$\mathbf{R}_{\mathrm{aj}}^{2} \%$} & \multirow{2}{*}{$S_{y x} \%$} \\
\hline & & & $\mathrm{B}_{0}$ & $\mathrm{~B}_{1}$ & & & \\
\hline \multirow{3}{*}{ NDVI } & 1 & 3 & $-32,3006$ & 44,3809 & $0,004^{* *}$ & 63,1 & 10,3 \\
\hline & 2 & 5 & $-23,0336$ & 32,6372 & $0,042^{* *}$ & 19,7 & 12,9 \\
\hline & 3 & $6-8$ & $-29,4532$ & 40,5279 & $0,003^{\star *}$ & 53,1 & 15,7 \\
\hline \multirow{3}{*}{ SRI } & 4 & 3 & $-5,68816$ & 0,98086 & $0,002^{* *}$ & 67,2 & 9,7 \\
\hline & 5 & 5 & $-3,81335$ & 0,76816 & $0,035^{\star *}$ & 21,3 & 12,7 \\
\hline & 6 & $6-8$ & $-5,44301$ & 0,93592 & $0,003^{* *}$ & 52,9 & 15,7 \\
\hline \multirow{3}{*}{ SAVI } & 7 & 3 & $-2,33423$ & 15,35661 & $0,023^{\star *}$ & 43,2 & 12,7 \\
\hline & 8 & 5 & $-0,83999$ & 9,9894 & $0,173^{\text {ns }}$ & 6,1 & 13,9 \\
\hline & 9 & $6-8$ & $-4,6714$ & 19,6933 & $0,008^{\star \star}$ & 44,1 & 17,1 \\
\hline
\end{tabular}

Fonte: Berger, 2014

${ }^{*} \mathrm{IAF}=\beta_{0}+\beta_{1} . \mathrm{IV}+\varepsilon_{\mathrm{i}} ;$ Prob $>\mathrm{F}=$ valor de significância; ${ }^{*}=$ Significativo para $\mathrm{F}=5 \%$; ns=não significativo para $\mathrm{F}=5 \% ; \mathrm{R}_{\mathrm{aj}}^{2} \%=$ Coeficiente de Determinação, em percentagem; $\mathrm{S}_{\mathrm{yx}} \%=$ Erro padrão da estimativa, em percentagem.

Em todas as idades analisadas o SRI mostrou melhor desempenho quando comparado aos demais IV, com exceção de ligeira superioridade do coeficiente de determinação $\left(R^{2}{ }_{\text {aj }} \%=53,1 \%\right)$ obtido com NDVI nas idades de seis e oito anos (Equação 3, Tabela 4). Aos três anos (Tabela 4), todas as equações mostraram melhores desempenhos em $\mathrm{R}_{\text {aj }}^{2} \%$ e $\mathrm{S}_{\mathrm{yx}} \%$ do que a respectiva análise geral (Tabela 3). A melhora dos ajustes nessa idade pode ser decorrente da maior cobertura foliar (maior IAF), o que diminui a mistura espectral (CHEN; CIHLAR, 1996). Entretanto, aos cinco anos, todas as equações mostraram pior desempenho em $\mathrm{R}_{\mathrm{aj}}^{2} \%$ e melhor dispersão entre os dados observados e estimados $\left(\mathrm{S}_{\mathrm{yx}} \%\right)$ em relação à equação geral por IV, à exceção do SRI que apresentou $\mathrm{S}_{\mathrm{yx}} \%$ ligeiramente superior $(12,7 \%)$.

Nas Figuras 2 a 4 podem ser observadas as relações entre o IAF e o NDVI, SRI e SAVI, e a distribuição dos resíduos, respectivamente, para todas as idades (a, b), e três (c, d), cinco (e, f) e seis-oito (g, h) anos.

Nas Figuras 2a, 3a e 4a podem ser observadas as relações gráficas ajustadas entre IAF e NDVI, SRI e SAVI, respectivamente. Todas as equações da Tabela 3 mostraram tendência em sobrestimar o IAF para valores inferiores a dois (Figuras $2 b, 3 b$ e $4 b$ ). Subestimativas de IAF foram observadas para valores de IAF superiores a 3,5 para NDVI e SRI, e superiores a três para SAVI. A equação ajustada por Flores et al. (2006) utilizando SRI subestimou os valores observados de IAF, o que foi justificado em parte pela presença de folhas mortas, que na verdade levariam a uma superestimativa do IAF medido no campo com o LAI-2000. 
Figura 2 - Relação entre o índice de área foliar do campo $\left(\mathrm{IAF}_{\mathrm{c}}\right)$ e o índice de vegetação da diferença normalizada (NDVI), obtido a partir de uma imagem Landsat 8/OLI, para (a) todas as idades e para $(c, e, g)$ três, cinco e seis-oito anos. IAF observado e estimado para (b) todas as idades e $(\mathbf{d}, \mathbf{f}, \mathbf{h})$ três, cinco e seis-oito anos.

Figure 2 - Relationship between ground leaf area index $\left(\mathrm{LAI}_{\mathrm{g}}\right)$ and the normalized difference vegetation index (NDVI), from a Landsat 8/OLI image, for (a) all the ages and (c, e, g) three, five and six-eight years. Observed and predicted LAI for (b) all the ages and $(\mathrm{d}, \mathrm{f}, \mathrm{h})$ three, five and six-eight years.

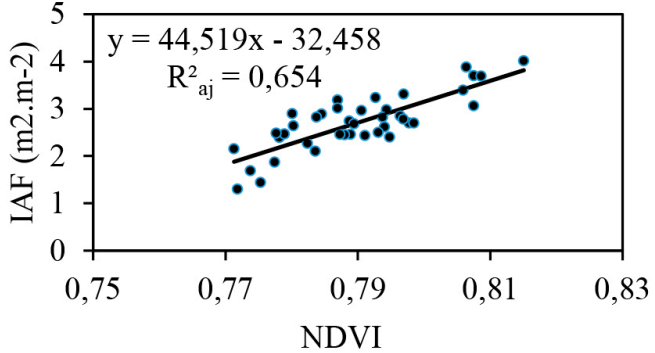

(a)

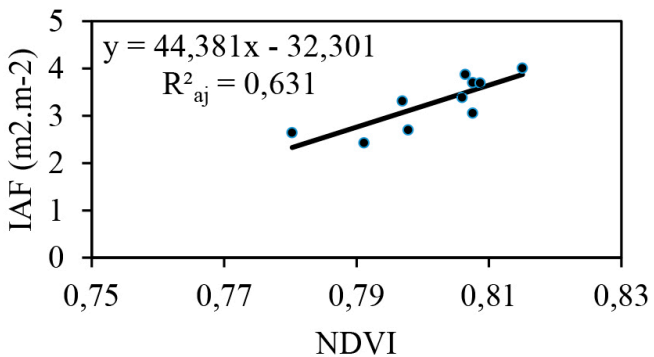

(c)

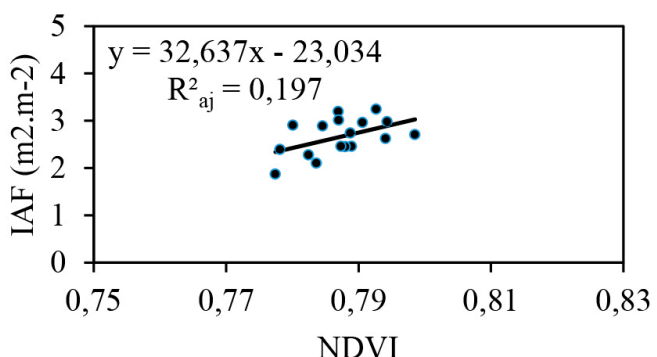

(e)

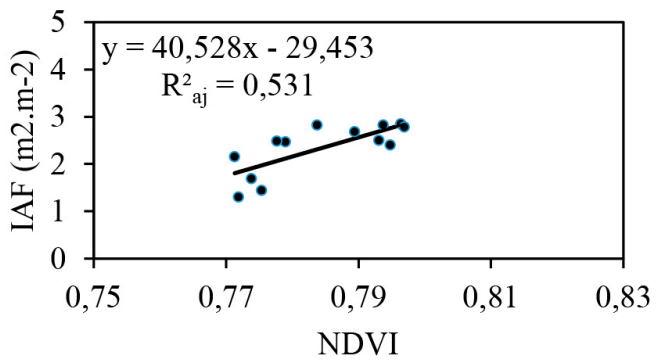

(g)

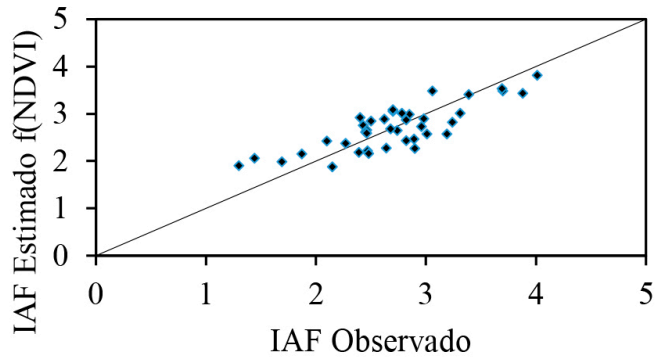

(b)

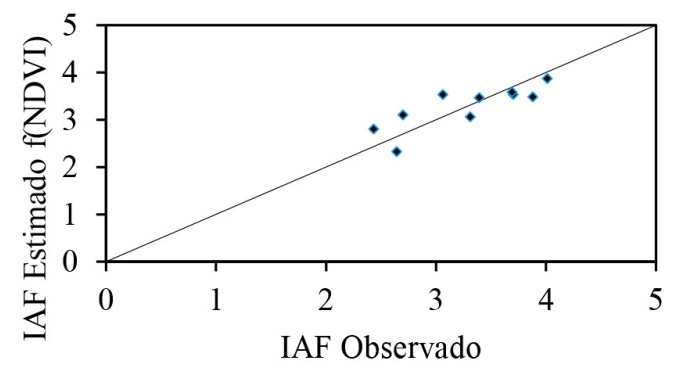

(d)

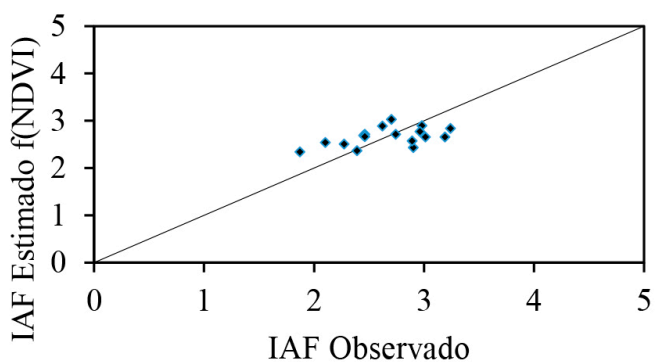

(f)

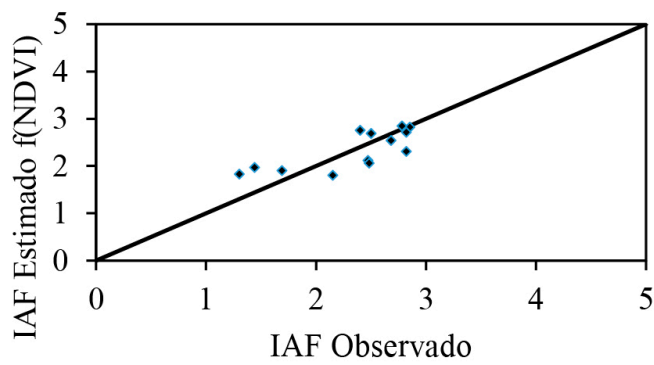

(h)

Fonte: Berger (2014) 
Figura 3 - Relação entre o índice de área foliar do campo $\left(\mathrm{IAF}_{\mathrm{c}}\right)$ e o índice da razão simples (SRI), obtido a partir de uma imagem Landsat 8/OLI, para (a) todas as idades e para (c, e, g) três, cinco e seis-oito anos. IAF observado e estimado para $(b)$ todas as idades e $(d, f, h)$ três, cinco e seis-oito anos.

Figure 3 - Relationship between ground leaf area index (LAI $)$ and the simple ratio index (SRI), from a Landsat 8/OLI image, for (a) all the ages and (c, e, g) three, five and six-eight years. Observed and predicted LAI for (b) all the ages and (d, f, h) three, five and six-eight years.

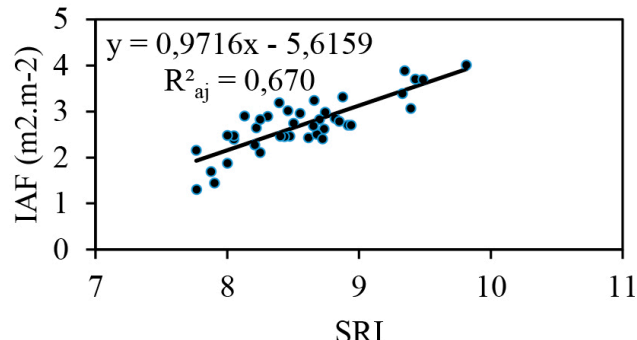

(a)

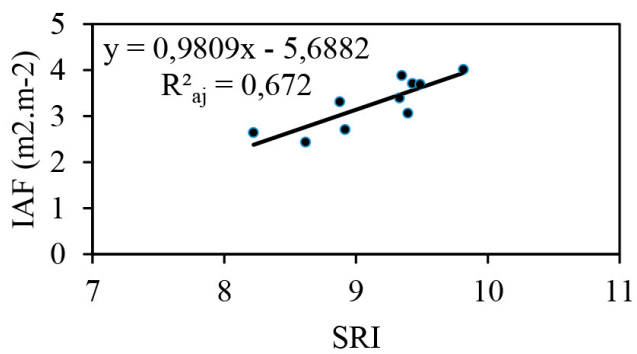

(c)

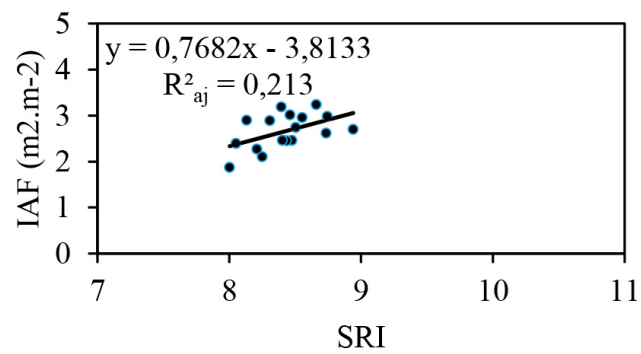

(e)

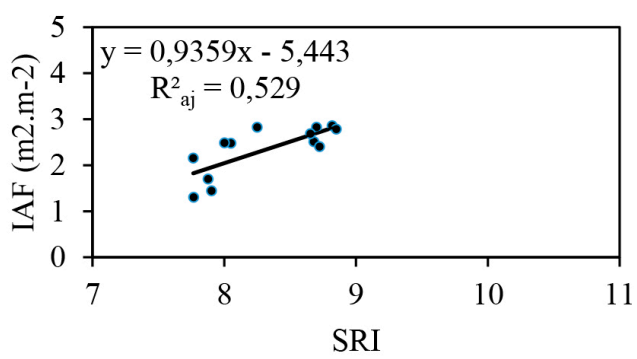

(g)

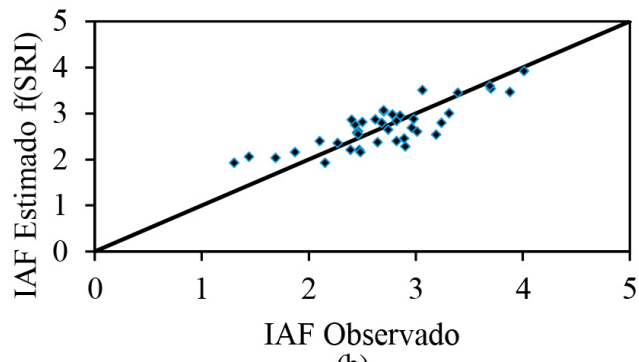

(b)

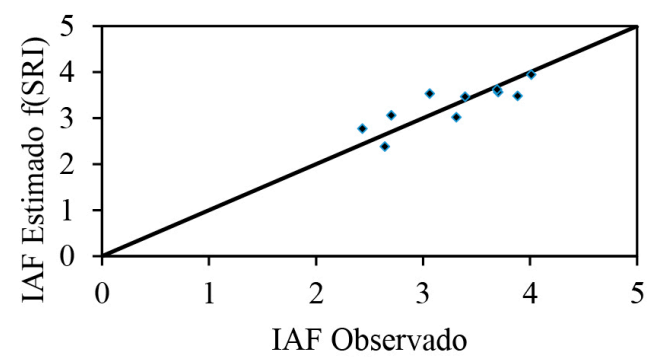

(d)

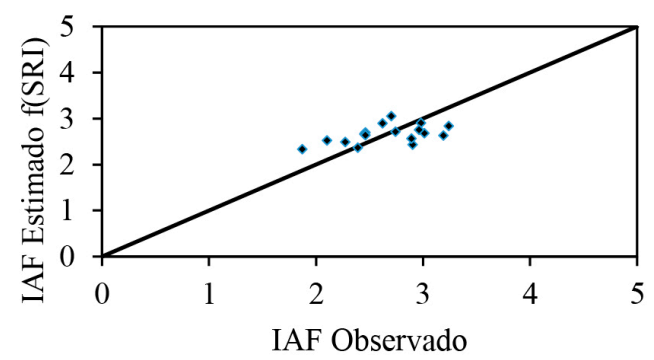

(f)

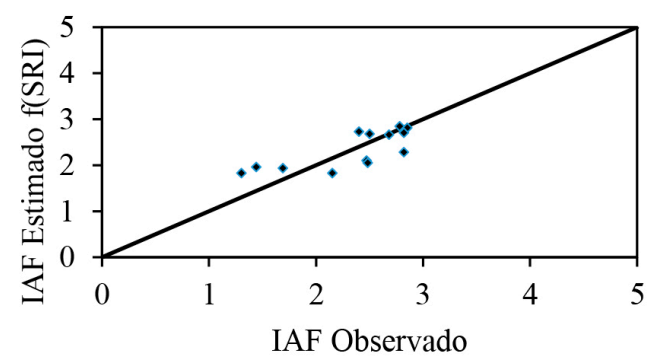

(h)

Fonte: Berger (2014) 
Figura 4 - Relação entre o índice de área foliar do campo (IAF ) e o índice de vegetação ajustado para o solo (SAVI), obtido a partir de uma imagem Landsat 8/OLI, para (a) todas as idades e para $(c, e, g)$ três, cinco e seis-oito anos. IAF observado e estimado para (b) todas as idades e $(d, f, h)$ três, cinco e seis-oito anos.

Figure 4 - Relationship between ground leaf area index $\left(\mathrm{LAI}_{\mathrm{g}}\right)$ and the soil adjusted vegetation index (SAVI), from a Landsat 8/OLI image, for (a) all the ages and (c, e, g) three, five and sixeight years. Observed and predicted LAI for (b) all the ages and $(\mathrm{d}, \mathrm{f}, \mathrm{h})$ three, five and six-eight years.

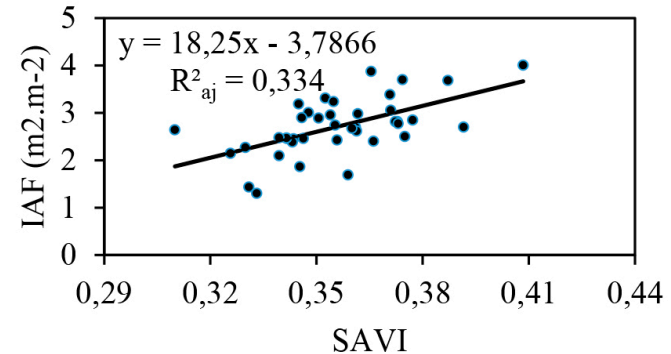

(a)

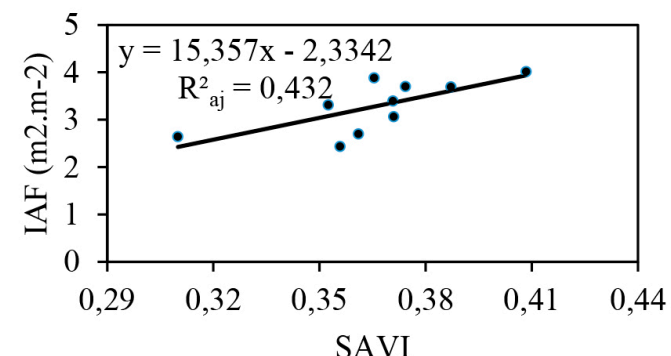

(c)

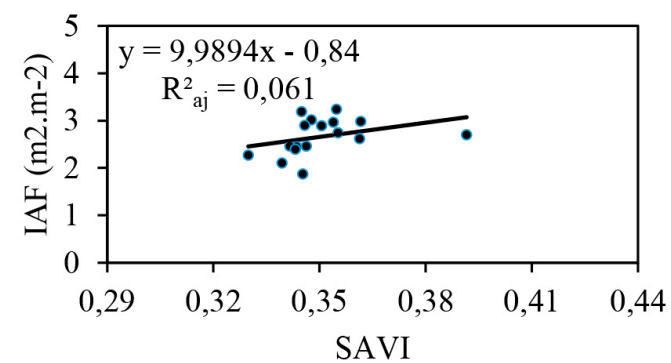

(e)

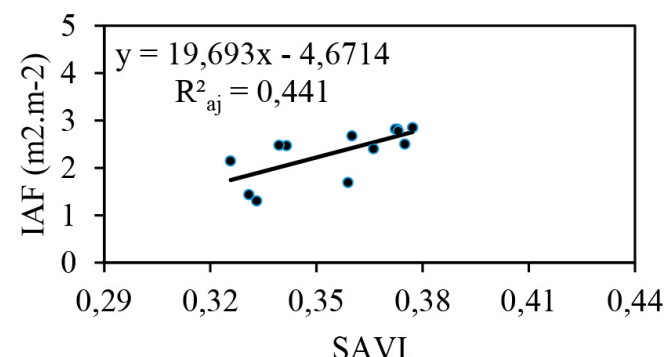

(g)

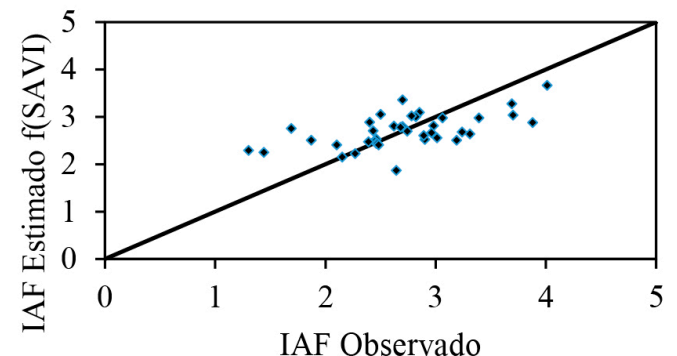

(b)

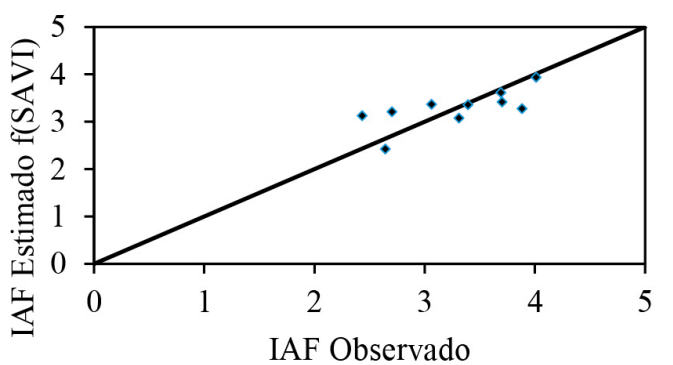

(d)

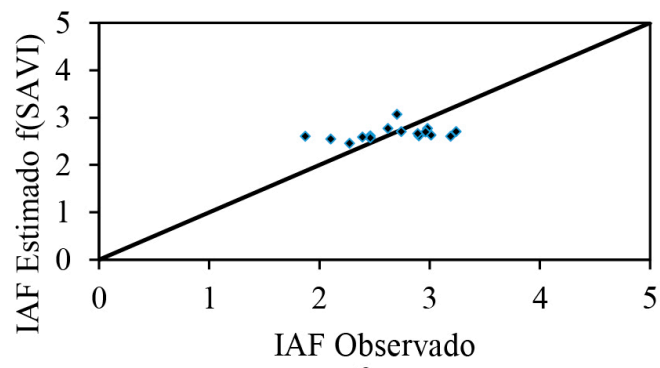

(f)

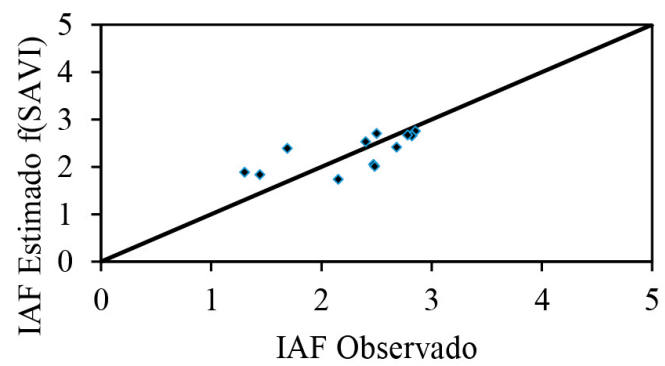

(h)

Fonte: Berger (2014) 
As Figuras 2c, 3c e 4c mostram a relação do IAF e o NDVI, SRI e SAVI aos três anos de idade, respectivamente. A equação para NDVI mostrou leve tendência em subestimar o IAF a partir de valores superiores a 3,5 (Figura 2d). Esta mesma tendência pode ser observada para SRI e SAVI, porém, de maneira mais sutil (Figuras $3 \mathrm{~d}$ e $4 \mathrm{~d}$ ).

Aos cinco anos, a equação ajustada para IAF em função de NDVI (Figura 2e) resultou em leve tendência de superestimar valores inferiores a 2,4 e subestimar valores superiores a 2,9 (Figura 2f). Este mesmo comportamento foi observado para IAF estimado por SRI (Figuras 3e, 3f). Para IAF em função de SAVI a equação ajustada (Figura 4e) mostrou-se tendenciosa para valores inferiores e superiores a 2,7 (Figura 4f).

Todas as equações ajustadas para as idades de seis e oito anos (Figuras $2 \mathrm{~g}$, $3 \mathrm{~g}$ e $4 \mathrm{~g}$ ) mostraram tendência em superestimar valores de IAF inferiores a dois (Figuras $2 \mathrm{~h}, 3 \mathrm{~h}$ e $4 \mathrm{~h}$ ).

Diante da pouco significativa melhora nos modelos ajustados especificamente para as diferentes idades avaliadas, optou-se por utilizar uma equação geral. Neste sentido, a equação 2 (Tabela 3) foi selecionada para estimar IAF em função de SRI, gerando a imagem IAF $_{S R}$ para o HFBN (Figura 5).

Figura 5 - Mapa do índice de área foliar estimado $\left(\mathrm{IAF}_{\mathrm{SR}}\right)$ pelo índice da razão simples (SRI), obtida a partir de uma imagem Landsat 8/OLI para o horto florestal Barba Negra (HFBN).

Figure 5 - Estimated leaf area index $\left(\mathrm{LAI}_{\mathrm{RS}}\right)$ by simple ratio index (SRI) from a Landsat 8/OLI image, for 'Barba Negra' farm.

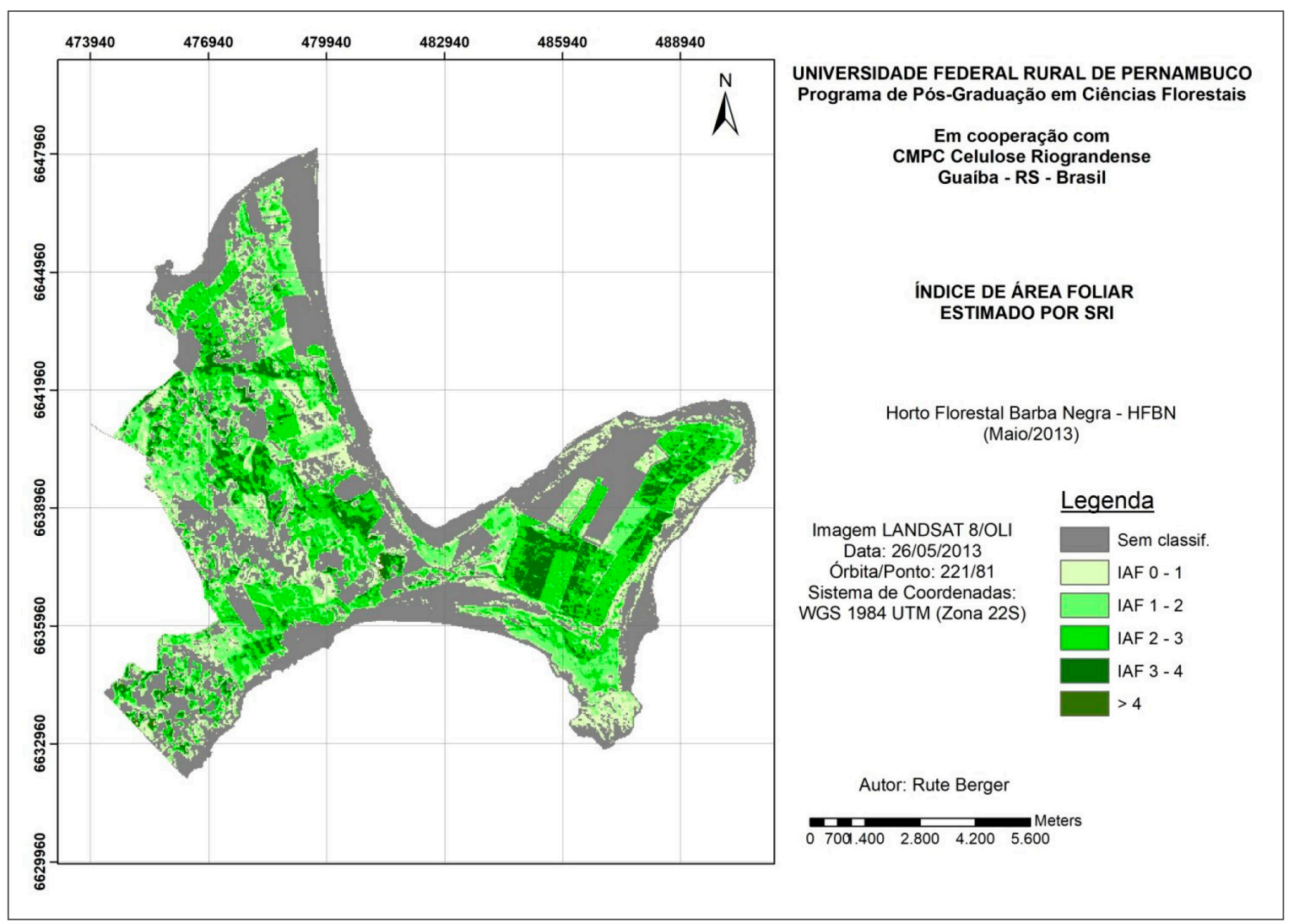

Fonte: Berger (2014) 
Chen e Cihlar (1996) comentam que a alta dispersão dos dados nas estimativas de IAF por IV normalmente é encontrada quando os pixels incluem uma combinação de reflectâncias originadas de diferentes tipos de vegetação (mistura espectral). Coberturas vegetais homogêneas, tais como os plantios florestais, geralmente apresentam uma forte relação entre o IAF e o IV correspondente. Neste sentido, imagens de alta resolução espacial tendem a oferecer melhores relações de IAF e IV do que as de menor resolução, pois essas podem conter misturas de diferentes tipos de cobertura (COLOMBO et al., 2003).

As variáveis que influenciam as propriedades espectrais da vegetação podem ter contribuído para produzir erros nas estimativas. Aspectos relacionados à topografia na geometria de iluminação (CANAVESI; PONZONI; VALERIANO, 2010; JENSEN, 2011), do posicionamento e da altura da vegetação em relação ao ângulo de elevação solar podem ter importantes impactos na absorção dos pigmentos das plantas. Jensen (2011) alerta para os fatores que podem influenciar na função de distribuição de reflectância bidirecional (BRDF) de um dossel, cujo comportamento anisotrópico interfere na energia incidente sobre este e, consequentemente, na energia refletida e captada pelo sensor remoto. Os fatores englobam, além dos mencionados anteriormente, as características dos alvos que podem influenciar na reflectância: vegetação-alvo, sub-bosque e solos (cor, textura, teor de umidade). Apesar da orientação sistemática das árvores nos talhões e da condição homogênea de espécies e idades, podem ocorrer outros fatores que influenciam na BRDF: forma e diâmetro das copas, IAF, número de árvores, falhas, entre outros. Esses fatores, aliados à maior resolução espacial do sensor podem mascarar a reflectância dos alvos.

Os erros também podem ser explicados pela relação entre a intensidade de amostragem e a extensão da área avaliada (SALVADOR; PONS, 1998). Nesses casos, os autores sugerem que os modelos ajustados por um grande número de amostras poderiam ter mais chances de incorporar causas de variação não controladas, tais como solos, clima, manejo, sub-bosque, entre outros, interferindo nas respostas espectrais de cada amostra.

Colombo et al. (2003) obtiveram melhores ajustes na estimativa de IAF ao utilizar a informação de textura em diferentes formações vegetais. Hernández et al. (2014), sugerem o uso e aperfeiçoamento de modelos híbridos ou de inteligência artificial para melhorar as predições de IAF em larga escala. Houborg, Anderson e Daughtry (2009) consideraram os modelos lineares (empíricos) como generalistas e propuseram um modelo baseado em processos (parâmetros biofísicos) para estimar o IAF por SRI, encontrando erros entre 10\% e 13\%. Esses resultados, porém, pouco diferem daqueles encontrados no presente estudo (12,5\%) e por Flores et al. (2006) (14\%), ajustando uma equação linear simples. Entretanto, o ajuste de modelos não lineares poderia contribuir para evitar efeitos indesejáveis e pouco realistas das estimativas de IAF $_{S R}$.

$\mathrm{Na}$ imagem IAF (Figura 5) mostra-se um panorama geral do comportamento do IAF estimado por sensoriamento remoto $\left(\mathrm{IAF}_{\mathrm{SR}}\right)$ em toda a extensão do HFBN. Podem ser observadas regiões (pixels) sem classificação, cujo $\mathrm{IAF}_{\mathrm{SR}}$ resultou ser inferior a zero, e regiões (pixels) com valores positivos de $\mathrm{IAF}_{\mathrm{SR}}$ (variando entre $>$ zero e $>4$ ), havendo um bom contraste entre setores com maior e menor $\mathrm{IAF}_{\mathrm{SR}}$. Os valores $\mathrm{IAF}_{\mathrm{SR}}$ negativo $(\mathrm{IAF}<0)$ podem ter sido ocasionados por várias características inerentes aos pixels, em maior ou menor proporção (mistura espectral), tais como influência dos solos, interferência atmosférica, propriedades espectrais dos alvos, bem como aos erros de ajuste da equação.

\section{Conclusão}

Os melhores ajustes resultaram da relação entre IAF e SRI, indicando que o IAF de plantios clonais de Eucalyptus saligna poderia ser estimado por produtos de sensoriamento remoto com uma precisão adequada. As análises por idade não melhoraram as estimativas, com exceção dos resultados obtidos aos três anos. Entretanto, todos os modelos ajustados mostraram tendência em subestimar e sobrestimar o IAF, o que indica que novos modelos matemáticos deveriam ser testados e maiores informações sobre o comportamento da resposta espectral devem ser consideradas. 


\section{Agradecimentos}

Os autores agradecem ao Programa de Pós-Graduação em Ciências Florestais da UFRPE, à Celulose Riograndense e à Forest Productivity Cooperative pelo apoio e disponibilidade dos dados.

\section{Referências}

ALMEIDA, A. C. et al. Mapping the effect of spatial and temporal variation in climate and soils on Eucalyptus plantation production with 3-PG, a process-based growth model. Forest Ecology and Management, Amsterdam, v. 259, p. 1730-1740, 2010.

ÁLVAREZ, J. et al. Factor influencing the growth of radiata pine plantations in Chile. Forestry, [s. l.], v. 86, p. 13-26, 2013.

ARIAS, D.; CALVO-ALVARADO, J.; DOHRENBUSCH, A. Calibration of LAI-2000 to estimate leaf area index (LAI) and assessment of its relationship with stand productivity in six native and introduced tree species in Costa Rica. Forest Ecology and Management, Amsterdam, v. 247, p. 185-193, 2007.

BERGER, R. et al. Utilización de índice de área foliar para optimización de inventários forestales. In: FOREST WORLD CONGRESS, 13., 2009, Buenos Aires. Proceedings [...]. Buenos Aires: FAO, 2009.

BERGER, R. Índice de área foliar estimado por ferramentas de sensoriamento remoto para a otimização de inventários de florestas plantadas. 2014. Tese (Doutorado em Ciências Florestais) - Universidade Federal Rural de Pernambuco, Recife, 2014.

BINKLEY, D. et al. Light absortion and use efficiency in forests: why patterns differ for trees and stands. Forest Ecology and Management, Amsterdam, v. 288, p. 5-13, 2013.

BULCOCK, H. H.; JEWITT, P. W. Spatial mapping of leaf area index using hyperspectral remote sensing of hydrological applications with a particular focus on canopy intersection. Hydrology and Earth System Sciences, [s. l.], v. 14, p. 383-392, 2010.

CANAVESI, V.; PONZONI, F. J.; VALERIANO, M. M. Estimativa de volume de madeira em plantios de Eucalyptus spp. Utilizando dados hiperespectrais e dados topográficos. Revista Árvore, Viçosa, MG, v. 34, n. 3, p. 539-549, 2010.

CANNELL, M. G. R. Physiological basis of wood production: a review. Scandinavian Journal of Forest Research, Stockholm, v. 4, p. 459-490, 1989.

CHEN, J. M.; CIHLAR, J. Retrieving leaf area index of boreal conifer forests using Landsat TM images. Remote Sensing of Environment, New York, v. 55, p. 153-162, 1996.

COLOMBO, R. et al. Retrieval of leaf area index in different vegetation types using high resolution satellite data. Remote Sensing of Environment, New York, v. 86, p. 20-131, 2003.

ENVI 5.1. Dowloads. [S. l.], 2014. Disponível em: http://www.envi.com.br/index.php/downloads. Acesso em: 26 maio 2014.

FAN, L. et al. Investigatin the relatioship between NDVI and LAI in semi-arid grassland in the Inner Mongolia using in-situ measurements. Theoretical and Applied Climatology, Wien, v. 95, p. 151-156, 2009.

FLORES, F. J. et al. Using multispectral satellite imagery to estimate leaf area and response to silvicultural treatments in loblolly pine stands. Canadian Journal of Forest Research, Ottawa, v. 36, p. 1587-1596, 2006. 
FOREST NUTRITION COOPERATIVE. Estimación de area foliar mediante el uso de imágenes satelitales Landsat 5. [S. l.], 2007. 15 p. (FNC Research Note, 33).

HERNÁNDEZ, C. et al. Data fusion for high spatial resolution LAI estimation. Information Fusion, [s. l.], v. 16, p. 59-67, 2014.

HOUBORG, R.; ANDERSON, M.; DAUGHTRY, C. Utility of an image-based canopy reflectance modeling tool for remote estimation of LAI and leaf chlorophyll content at the field scale. Remote Sensing of Environment, New York, v. 113, p. 259-274, 2009.

HUETE, A. R. A soil-adjusted vegetation index (SAVI). Remote Sensing of Environment, New York, v. 25, p. 295-309, 1988.

HUETE, A.R.; JACKSON, R.D.; POST, D.F. Spectral response of a plant canopy with different soil backgrounds. Remote Sens. Environ., [s.1.], v. 17, p. 37-53. 1985.

JACKSON, R. D.; HUETE, A. R. Interpreting vegetation indices. Preventive Veterinary Medicine, Amsterdam, v. 11, p. 185-200, 1991.

JENSEN, J. R. Sensoriamento remoto da vegetação. In: JENSEN, J. R. Sensoriamento remoto do ambiente: uma perspectiva em recursos terrestres. 2. ed. São José dos Campos: Parêntese, 2011. Cap. 11, p. 357-410.

JONCKHEERE, I. et al. Review of methods for in situ leaf area index determination. Part I: Theories, sensors and hemispherical photography. Agricultural and Forest Meteorology, Amsterdam, v. 121, p. 19-35, 2004.

LE MAIRE, G. et al. Leaf area index estimation with MODIS reflectance time series and model inversion during full rotations of Eucalyptus plantations. Remote Sensing of Environment, New York, v. 115, p. 586-599, 2011.

LI-COR. LAI-2000 Plant canopy analyser. Lincoln: LI-COR Inc., 1992. 198 p.

PEEL, M. C.; FINLAYSON, B. L.; MCMAHON, T. A. Updated world map of the Köppen-Geiger climate classification. Hydrology and Earth System Sciences, [s. l.], v. 11, p. 1633-1644, 2007.

PONZONI, F. J.; SHIMABUKURO, Y. E. Sensoriamento remoto no estudo da vegetação. São José dos Campos: Parêntese, 2010. 127 p.

RODY, Y. P. et al. Estimates of the leaf area index (LAI) using LAI-2000 and hemispherical photos in Eucalyptus plantations. Ciência Florestal, Santa Maria, v. 24, n. 4, p. 923-932, 2014.

SALVADOR, R.; PONS, X. On the applicability of Landsat TM images to Mediterranean forest inventories. Forest Ecology and Management, Amsterdam, v. 104, p. 193-208, 1998.

SONG, C. Optical remote sensing of forest leaf area index and biomass. Progress in Physical Geography, London, v. 37, n. 1, p. 98-113, 2013.

UNITED STATES GEOLOGICAL SURVEY. Landsat 8. [S. l.], 2013. Disponível em: http:// earthexplorer.usgs.gov/. Acesso em: 17 out. 2013.

XAVIER, A. C.; SOARES, J. V.; ALMEIDA, A. C. Variação do índice de área foliar de eucalipto ao longo de seu ciclo de crescimento. Revista Árvore, Viçosa, MG, v. 26, n. 4, p. 421-427, 2002. 